Reprod. Nutr. Dévelop., 1987, 27 (4), 817-827.

\title{
Rôle des cellules B de l'intestin moyen chez Centropages typicus (Copepoda, Calanoida)
}

\author{
J. ARNAUD, M. BRUNET, J. MAZZA
}

Laboratoire de Biologie animale, Case 18, Université de Provence, Place Victor-Hugo, 13331 Marseille Cedex 3, France.

Summary. Role of midgut B-cells in Centropages typicus (Copepoda, Calanoida).

The role of B-cells was studied using both cytochemical and cytophysical techniques (polysaccharide and protein detection; use of a tracer and microanalysis investigations by $X$ ray spectrographyl. These techniques demonstrated : a) An intensive endocytose activity during which the very numerous apical vesicles and vacuoles are labelled by the Thiéry's test and contain ferritin. b) The presence of sulphur (mainly in the form of sulphated substrates) and aluminum, detected by microanalysis from sections through lysosomal material of vacuoles and dense bodies. These findings suggest that $B$-celles might exert a double function dealing with an intracellular digestive process and an excretion-detoxication mechanism.

\section{Introduction.}

Au cours de recherches antérieures sur l'intestin moyen des copépodes calanoides, nous avons analysé les caractères ultrastructuraux des principaux types cellulaires de l'épithélium digestif (Arnaud et al., 1978, 1980). Les cellules B représentent la catégorie la plus remarquable et ressemblent étroitement aux cellules de même nom décrites au niveau de l'hépatopancréas des décapodes (Loizzi, 1971). Elles montrent en effet dans ces deux phylums la formation d'un appareil vacuolaire, édifié à partir de vésicules d'endocytose. Chez le copépode Centropages typicus, nous avons avancé que cet appareil, alimenté en phosphatases et en arylsulfatases, est assimilable à un système lysosomal où se déroule une phase digestive intracellulaire (Arnaud et al., 1984). Par ailleurs, d'autres observations, notamment l'extrusion des cellules $B$ dans la lumière intestinale à la fin de leur évolution, suggèrent également pour elles un rôle excréteur. Aussi, dans le cadre de la poursuite de nos investigations sur l'épithélium digestif, nous sommes-nous attachés à préciser le fonctionnement des cellules $B$ en utilisant conjointement des techniques cytochimiques (détection de protéines et de polysaccharides) et cytophysiques (emploi de traceur - ferritine - et microanalyse par spectrographie des rayons $\mathrm{X}$ ). 


\section{Matériel et méthodes.}

1. Expérimentation à l'aide de ferritine. - Les expériences sont réalisées sur des spécimens de Centropages typicus prélevés dans le golfe de Marseille, triés au laboratoire et laissés jusqu'au lendemain dans de l'eau de mer filtrée, sans nourriture. Des lots de 7 à 8 copépodes sont alors répartis dans des erlenmeyers contenant $10 \mathrm{ml}$ d'un milieu nutritif algal et $10 \mathrm{mg}$ de ferritine. Au bout de $30 \mathrm{~min}$ à $1 \mathrm{~h}$ les copépodes sont prélevés et fixés pour l'étude en microscopie électronique selon les modalités citées antérieurement (Arnaud et al., 1980), I'inclusion étant faite au spurr.

2. Etude cytochimique. - Recherche de polysaccharides : des coupes fines d'animaux prélevés en mer et inclus à l'araldite après double fixation sont traitées selon la technique PATAg (acide périodique-thiocarbohydrazide TCH ou thiosemicarbazide TSC - protéinate d'Ag) (Thiéry et Rambourg, 1974) ; les durées du traitement par la TCH ou la TSC ont été de $40 \mathrm{~min}, 5 \mathrm{~h}, 24 \mathrm{~h}, 48 \mathrm{~h}, 60 \mathrm{~h}$ et $72 \mathrm{~h}$; les contrôles correspondent à des coupes subissant le même traitement mais oxydées par une solution à $7 \% d^{\prime} \mathrm{H}_{2} \mathrm{O}_{2}$ à 110 volumes ; des coupes ont également été observées après oxydation seule.

Action de protéases : les coupes proviennent d'animaux inclus à l'épon après double fixation ; les solutions d'enzymes et les modalités de leur utilisation et de contrôle sont identiques à celles employées antérieurement (Arnaud et al., 1982).

3. Microanalyse par spectrographie des rayons $\times\left({ }^{1}\right)$. - Une recherche qualitative d'éléments minéraux a été entreprise sur des coupes de spécimens inclus à l'araldite après double fixation. Des coupes de 0,2 à $0,3 \mu \mathrm{m}$ d'épaisseur et montées sur grille sont étudiées grâce à un système de microanalyse X Tracer N 2000 par dispersion d'énergie, adapté sur un microscope électronique à transmission Philips EM $400 \mathrm{~T}$; le spot du faisceau de sonde a un diamètre de $50 \AA$. D'autres coupes de 3 à $5 \mu \mathrm{m}$ d'épaisseur sont étalées sur des plots en carbone, métallisées au carbone et étudiées grâce au même système adapté sur un microscope électronique à balayage Jeol JSM 35 CF ; le faisceau de sonde est ponctuel (diamètre de $300 \AA$ l ou concerne une fenêtre de $4,5 \times 4,5 \mathrm{~cm}$.

\section{Résultats.}

1. Expérimentation à l'aide de ferritine. - La ferritine se retrouve dans la lumière du tube digestif des copépodes, plus ou moins mélangée à des algues (fig. 2). Des individus nourris pendant 45 min montrent une endocytose très nette

(') Nous remercions M. Passerel pour son assistance technique lors de cette étude réalisée au Centre Interuniversitaire de Microscopie Electronique et de Microanalyse des universités d'Aix-Marseille II et III. 
de ferritine au niveau des cellules B : celle-ci s'observe au voisinage du plasmalemme, dans les vésicules de pinocytose en formation, dans la plupart des vésicules et petites vacuoles apicales et même dans les vacuoles plus grandes, subapicales et médianes (fig. 1, 2). Les plans de coupes favorables indiquent que la ferritine est localisée préférentiellement près de la membrane des vésicules endocytotiques.
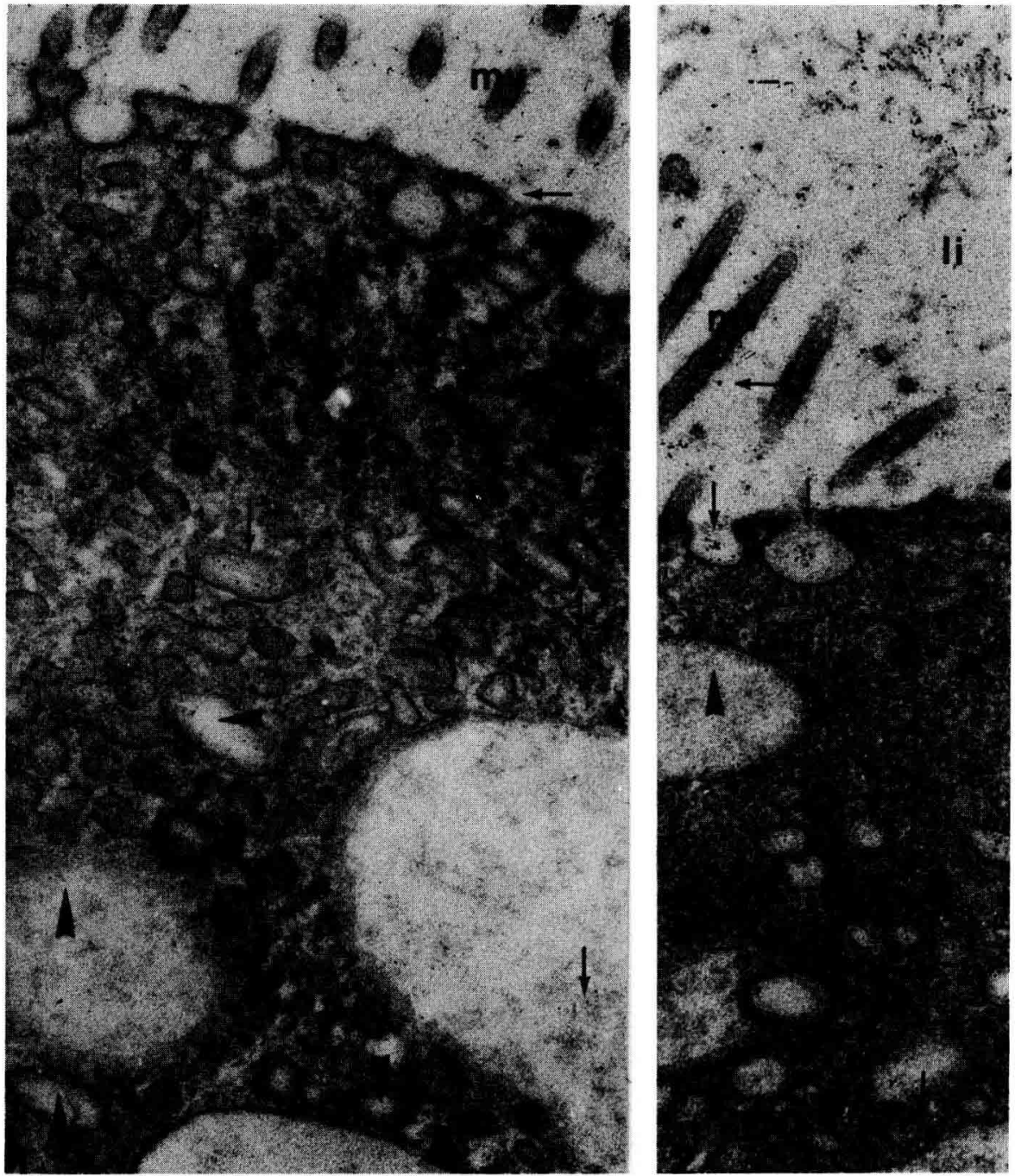

FIG. 1-2. - Portions apicales de 2 cellules $B$ montrant l'endocytose de territıne. Noter la présence de ferritine (flèches) dans la plupart des vésicules apicales et sa localisation près de la membrane dans les vésicules internalisées (triangles). (li = lumière intestinale; $m v=$ microvillosités). $\times 35000$. 
2. Cytochimie. - Les tests PATAg fournissent des résultats positifs même avec de courtes durées d'incubation dans la TCH ou la TSC; pour des temps prolongés, le marquage ne s'accentue que légèrement. Ce marquage affecte la membrane apicale, les membranes de toutes les vésicules et vacuoles, le matériel intravacuolaire, quelle que soit sa densité, et tous les types de corps denses (fig. 3, 4). Chez les témoins, il n'y a aucun marquage des membranes et du contenu fibrillaire des vacuoles; mais, fréquemment, du matériel intravacuolaire en amas et des inclusions denses structurées montrent des dépôts argentiques (fig. 5), parfois aussi importants que ceux des tests. Enfin des contrôles soumis à une oxydation seule peuvent présenter encore des structures contrastées en raison d'une élimination probablement incomplète de l'osmium.

L'action des trois protéases utilisées (pepsine, pronase, trypsine) est peu différente, quoique celle de la trypsine semble plus faible. Elles provoquent un éclaircissement partiel des amas denses déposés dans les grandes vacuoles (fig. 11) ; les corps denses proprement dits subissent soit un éclaircissement généralisé et prononcé (corps denses homogènes) (fig. 7), soit un éclaircissement périphérique ménageant une zone centrale granuleuse (corps denses structurés) (fig. 10, 12). Chez les témoins exposés seulement à l'action du solvant protéique, toutes ces structures subissent également un éclaircissement, mais moins important que dans les tests (fig. 8, 9).

3. Microanalyse par spectrographie des rayons $X$. - L'étude en microanalyse permet de détecter dans les corps denses des cellules $B$ la présence fréquente d'aluminium et de soufre, plus rarement de calcium (fig. 13a) ; ces minéraux peuvent apparaître aussi au niveau des zones peu denses du contenu vacuolaire. Les résultats sont toujours significatifs sur les coupes de 3 à $5 \mu \mathrm{m}$ analysées en balayage ; ils sont plus faibles à nuls sur les coupes de 0,2-0,3 $\mu \mathrm{m}$ analysées en transmission, bien que les condensations de matériel intravacuolaire soient importantes et fréquentes, comme le montrent les clichés réalisés sur ces coupes (fig. 14). Par comparaison, le contenu des zones intermédiaire et postérieure de l'intestin moyen montre la présence constante des mêmes éléments (fig. 13b), auxquels peuvent s'ajouter un peu de chlore et de potassium. Des analyses effectuées sur un calanoide d'eaux temporaires de Camargue, Mixodiaptomus kupelwieseri, fournissent des résultats tout à fait analogues ; le pic de $\mathrm{Ca}$ est généralement plus élevé (fig. 13c-d) et du fer est parfois présent au niveau du contenu intestinal.

FIG. 3-5. - Portions de cellules $B$ après test PATAg sans contrastage. Fig. 3-4. Test PATAg lacide periodique : $25 \mathrm{~min}$; triocarbohydrazide : $60 \mathrm{~h}$; protéinate d'argent : $30 \mathrm{~min}$ ). Noter le marquage du plasmalemme, des membranes de toutes les vésicules et petites vacuoles, du matériel intravacuolaire fibrillaire (flèche longue) et de corps denses (triangles) montrant parfois un noyau central (astérisque) moins réactif que la couche de matériel périphérique. Remarquer le contenu vacuolaire d'aspect similaire au matériel présent au contact de la membrane des vacuoles (flèches courtes). Fig. $3: \times 14000$; fig. $4: \times 40000$. Fig. 5. Contrôle PATAg loxydation par $\mathrm{H}_{2} \mathrm{O}_{2}: 15$ min ; thiocarbohydrazide : $72 \mathrm{~h}$; protéinate d'Ag : $30 \mathrm{~min}$ ). Noter l'absence de réaction au niveau des vésicules apicales et de la plupart des vacuoles ; cependant le matériel de certaines vacuoles offre une réactivité au protéinate d'argent (flèches). $\times 9000$. Les contrôles effectués après une oxydation pendant 20 min et 25 min par $\mathrm{H}_{2} \mathrm{O}_{2}$ fournissent des clichés analogues. 

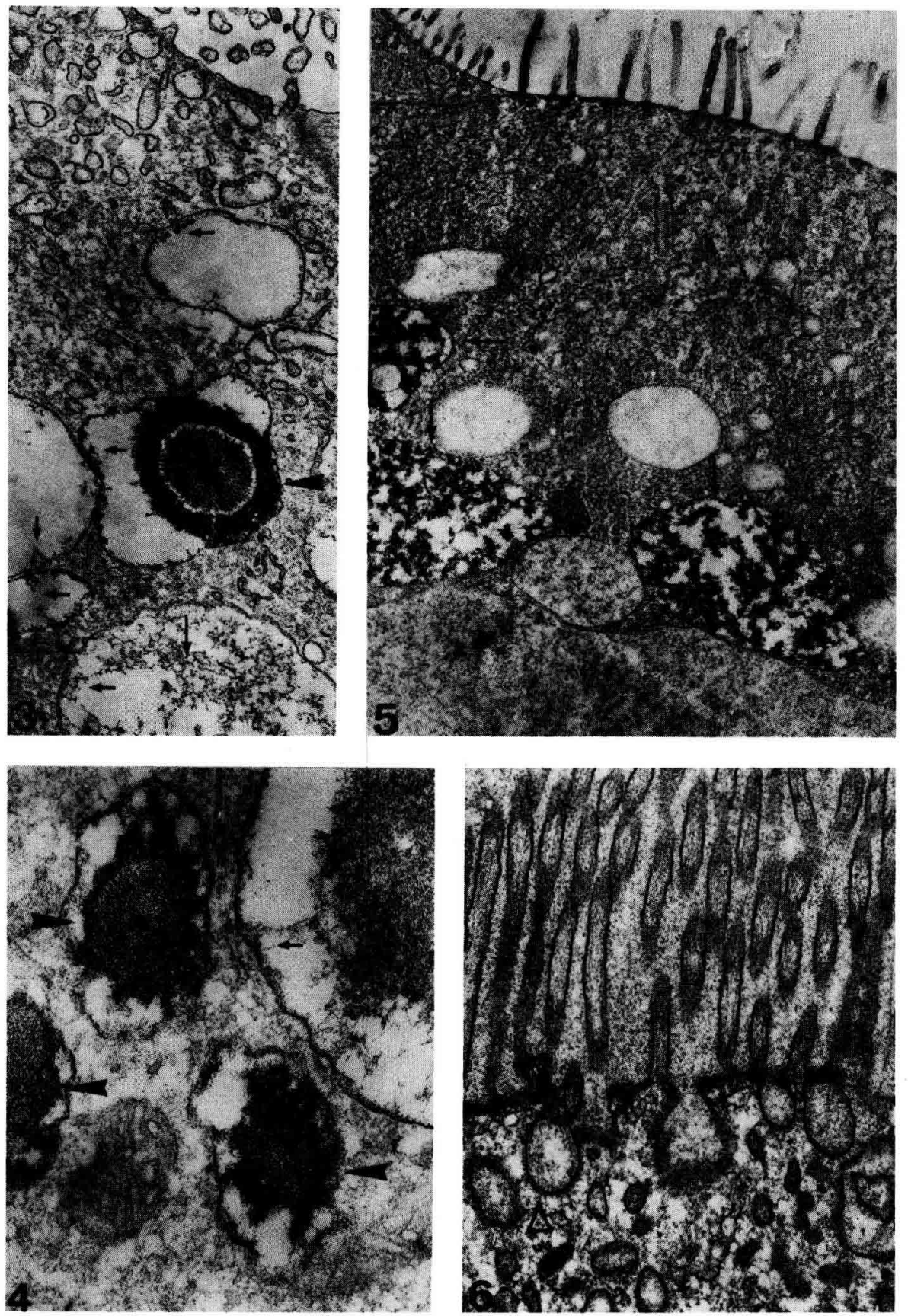

FIG. 6. - Portion apicale d'une cellule $B$ lcoupe contrastée standard obtenue après double fixation glutaraldéhyde $-\mathrm{OsO}_{4}$ et inclusion à l'araldite). Noter le feutrage important entre les microvillosités et dans les vésicules de pinocytose et la présence d'un revêtement externe bien visible au niveau d'une vésicule en formation (triangle) suggérant l'intervention de clathrine lors de l'endocytose. $\times 21000$. 

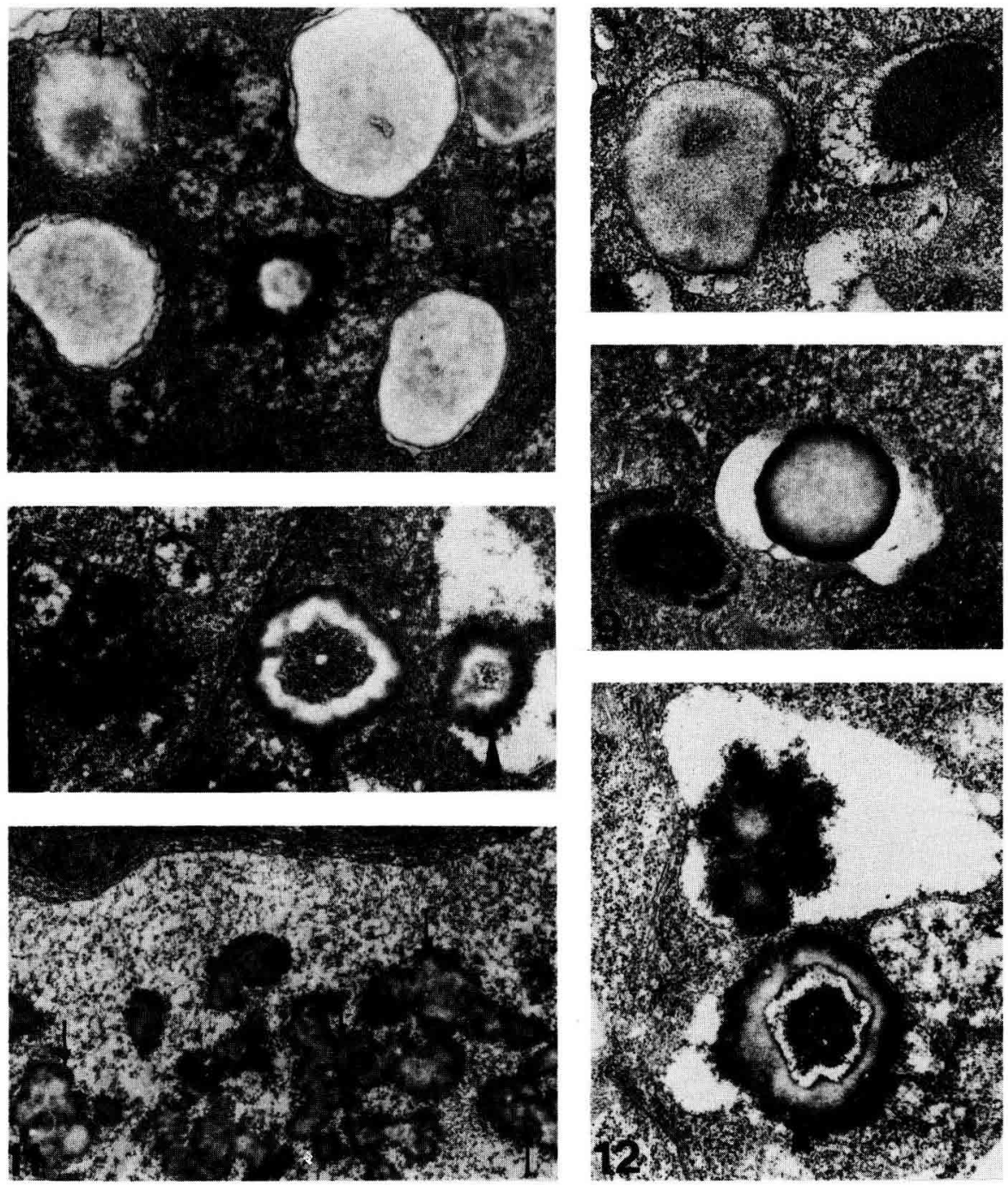

FIG. 7-12. - Tests de digestion enzymatique par des protéases. Fig. 7. Portion de cellule B après action d'une solution de pepsine $0,5 \%$ pendant $13 \mathrm{~h}$ : les corps denses (flèches) sont éclaircis à des degrés divers alors que le matériel moins concentré de vacuoles n'est pas altéré, sauf localement (triangle) au niveau d'une zone plus dense. $\times 9300$. FIG. 8-9. - Portions de cel/ules $B$ après action du solvant de la pepsine seul pendant $12 \mathrm{~h}$ : des corps denses (flèches) sont également éclaircis. Fig. $8: \times 9400 ;$ fig. $9: \times 8000$. FIG. 10-12. - Portions de cellules $B$ après action d'une solution de trypsine $0,5 \%$ (fig. 10-11) et d'une solution de pronase $0,5 \%$ (fig. 12) pendant $12 \mathrm{~h}$ : action similaire à celle de la pepsine, mais un peu moins forte pour la trypsine ; seule la couche périphérique des corps denses structurés (triangles) est éclaircie ; dans les vacuoles, le matériel est peu altéré sauf au niveau des foyers de concentration (flèches) ; chez les témoins, les structures denses subissent également un éclaircissement, mais celui-ci est généralement moins marqué que dans les tests. Fig. $10: \times 9000 ;$ fig. $11: \times 6600 ;$ fig. $12: \times 9900$. 

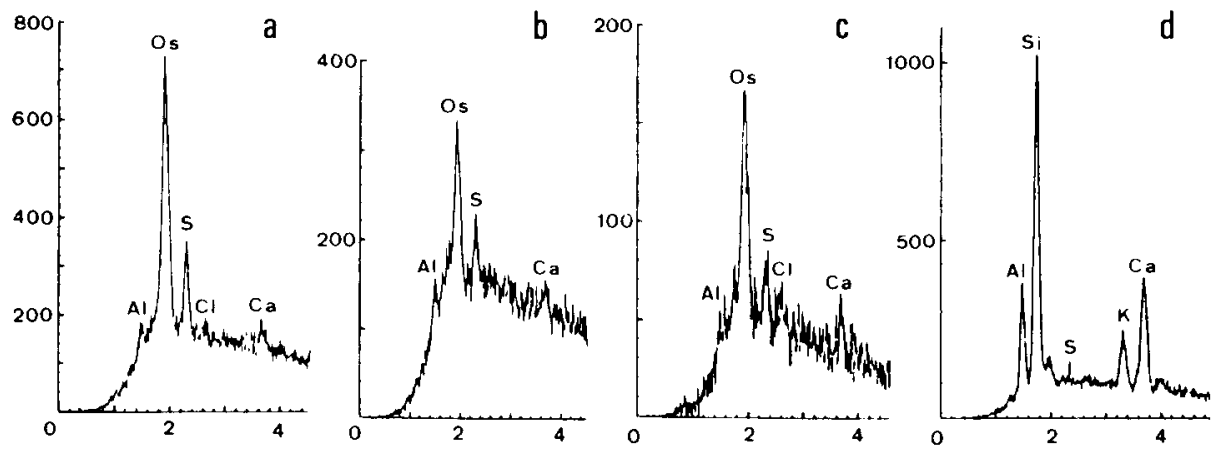

13

FIG. 13. - Microanalyse par spectrographie des rayons $X$. Fig. 13a-b : microanalyse au niveau d'un corps dense d'une cellule $B$ de l'épithélium intestinal (a) et du contenu luminał (b) chez Centropages typicus. Fig. 13c-d : microanalyse au niveau d'un corps dense d'une cellule B de l'épithélium intestinal (c) et du contenu luminal (d) chez Mixodiaptomus kupe/wieseri. Dans ce dernier cas, le pic de Si peut s'expliquer par la présence de frustules de diatomées et (ou) par celle de particules provenant du couteau de verre utilisé pour la réalisation des coupes. En abscisses : nombre de coups; en ordonnées : énergie de rayonnement en $\mathrm{KeV}$; temps de lecture : $100 \mathrm{~s}$.

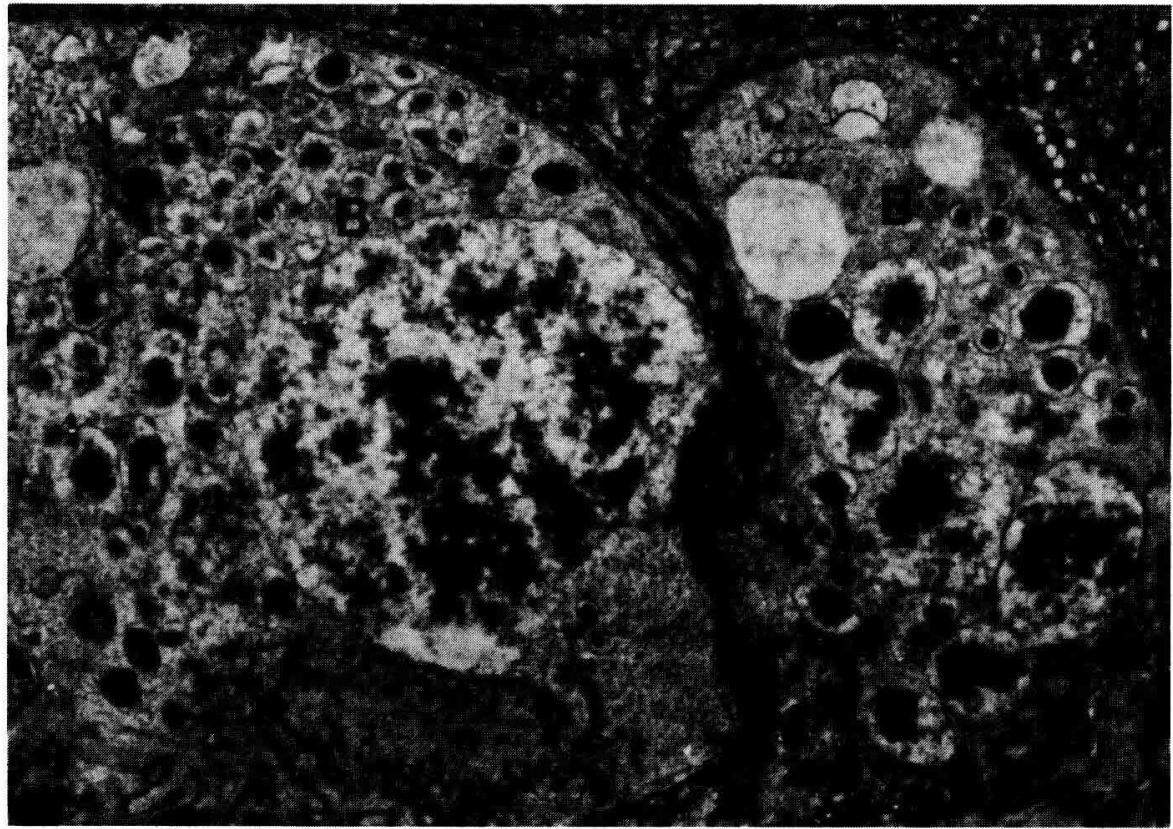

FIG. 14. - Coupe épaisse $(0,3 \mu \mathrm{m})$ non contrastée réalisée au niveau de l'épithélium intestinal de Centropages typicus. Noter les très nombreuses condensations de matériel dans le système vacuolaire des cellules $B$. ( $n$ : noyau). $\times 2760$. 


\section{Discussion.}

1. Activité endocytotique. - Les résultats des tests de Thiéry et de l'expérimentation à la ferritine confirment que les cellules $B$ sont le siège d'une activité endocytotique intense : en effet, toutes les structures vésiculaires présentes à l'apex sont marquées après le test de Thiéry et, par ailleurs, presque toutes recèlent de la ferritine. L'activité d'endocytose aboutit à la formation du système vacuolaire des cellules $B$; le marquage très sélectif au test de Thiéry de la membrane plasmique apicale, des membranes des vésicules de pinocytose et des différents éléments de ce système tend à prouver que celui-ci résulte, pour l'essentiel, d'une coalescence des vésicules de pinocytose. Nos données appuient celles d'AlMohanna et al. (1985) sur les cellules B de Penaeus semisulcatus (décapode), acquises en utilisant différents traceurs ; par contre, elles diffèrent sensiblement de celles obtenues par Durfort (1981) pour le copépode d'eau douce Cyclops strenuus chez lequel de nombreuses vésicules tubulaires apicales, non réactives au test de Thiéry, représenteraient du réticulum endoplasmique lisse.

Nos résultats tendent aussi à montrer qu'une membranogenèse à partir de certaines vésicules apicales semble insignifiante dans ces cellules; la conséquence serait à terme un déficit membranaire apical expliquant l'aspect conique fréquent des volumineuses cellules $B$ âgées et, finalement, leur extrusion dans la lumière intestinale.

2. Origine et nature du contenu vacuolaire. - Lors de la pinocytose, des molécules provenant du contenu intestinal doivent être incorporées dans les cellules B puis soumises à l'action d'hydrolases intracellulaires telles que phosphatase acide et arylsulfatase, déchargées dans le système vacuolaire de nature lysosomale (Arnaud et al., 1984). Le glycocalyx joue probablement un rôle dans ce phénomène bien qu'il soit peu apparent sur les clichés obtenus avec les spécimens d'expérimentation. Par contre, un feutrage important s'observe parfois nettement au niveau apical des cellules $B$ sur des coupes de copépodes préparés dans des conditions standard (fig. 6); ce feutrage, qui se retrouve dans les vésicules d'endocytose, représente sans doute le glycocalyx et les substances piégées ayant pour origine des aliments et peut-être des résidus des sécrétions provenant des glandes labrales, qui jouent un rôle digestif important (Arnaud et al., 1981). On peut donc penser que ces substances auxquelles s'ajoutent les enzymes lytiques, et éventuellement la fraction polysaccharidique des glycoprotéines membranaires appartenant au glycocalyx, contribuent dans un premier temps à la naissance d'un matériel vacuolaire dispersé, finement fibrillaire ; sa réactivité rapide et forte au PATAg (supprimée chez les témoins) indique qu'il s'agit de polysaccharides ou de glycoprotéines aisément accessibles lors du test. Ce matériel doit se transformer progressivement en corps denses plus ou moins structurés, produits du catabolisme vacuolaire. Ces formations résiduelles ne sont pas élaborées à partir de structures myéliniques, très rarement observées dans les cellules $B$; elles contiennent une fraction protéique assez faible d'après les tests de digestion enzymatique et probablement une fraction polysaccharidique (cf. ci-dessous) ; mais, 
comme la plupart sont réactives dans les contrôles PATAg en raison sans doute de la persistance d'osmium après oxydation, on ne peut conclure avec certitude à la présence d'hydrates de carbone à partir des tests effectués.

3. Rôle excréteur des cellules $B$. - La présence d'éléments minéraux dans les inclusions denses principalement indiquerait une phénomène de concentration intralysosomale, déjà observé chez d'autres invertébrés (Chassard-Bouchaud et Galle, 1986 ; Martoja et Martoja, 1973 ; Jeantet et al., 1980 ; Mason et Simkiss, 1984), des crustacés décapodes, notamment (Gibson et Barker, 1979 ; ChassardBouchaud, 1981 ; Chassard-Bouchaud et Galle, I.c. ; Lyon et Simkiss, 1984). De telles inclusions lysosomales, assurant essentiellement une fonction d'excrétion et de détoxication, comprennent toutes les formations originales signalées sous le nom de " grains pigmentés " ou " inclusions brunes " et se rencontrent dans des cellules d'organes divers (cœur, intestin et diverticules intestinaux, hépatopancréas, organes excréteurs, tissu conjonctif). Dans l'hépatopancréas des décapodes, ces inclusions n'étaient signalées qu'au niveau des cellules $R$, mais elles viennent d'être détectées aussi dans les vacuoles des cellules $B$ au cours du cycle digestif de Penaeus semisulcatus (Al-Mohanna et Nott, 1986). Par ailleurs, l'aspect structuré de certaines inclusions chez Centropages typicus rappelle celui des concrétions minérales de divers types décrites chez de nombreux invertébrés, souvent désignées sous le nom de sphérites ou sphérocristaux et ne constituant parfois que des formes particulières d'inclusions lysosomales. Ces concrétions diffèrent par leur origine - réticulum, le plus souvent, mais aussi lysosomes, mitochondries, espace extra-cellulaire - et leur rôle - réserves minérales, régulation ionique, détoxication et excrétion - . Elles se distinguent des inclusions précédentes par leur mode de formation, résultant d'un dépôt en couches concentriques d'éléments minéraux sur un ligand organique, mucosubstances acides ou métallothionéines dans le cas d'exposition à des métaux lourds, comme l'attestent les travaux réalisés chez les décapodes (Graf et Michaut, 1977 ; Gibson et Barker, 1.c., Chassard-Bouchaud, 1981, 1982) et chez divers autres invertébrés (Martoja et Martoja, I.c. ; Humbert, 1978 ; Jeantet et al., l.c. ; Durfort, 1982b). Chez les copépodes, les seuls travaux relatifs à des inclusions minéralisées concernent Cyclops strenuus (Durfort, 1981, 1982a) : des concrétions volumineuses, de type sphérocristaux, prennent naissance dans des vésicules de réticulum et comprennent une matrice polysaccharidique riche en $\mathrm{Ca}, \mathrm{K}$ et $\mathrm{Mg}$; elles joueraient un rôle de stockage des sels minéraux utilisés dans la consolidation du tégument au cours des mues post-larvaires. Chez Centropages typicus, le soufre détecté est dû probablement à une accumulation vacuolaire, intralysosomale, d'une matrice organique sulfatée résiduelle, capable de piéger divers ions, aluminium et calcium dans le cas présent. La détection histochimique de mucosubstances sulfatées (MPS) dans les cellules B (Arnaud et al., 1984) appuie cette hypothèse. Ce soufre aurait une origine endogène, comme dans les concrétions ou les inclusions dans lesquelles il est signalé (Humbert I.c. ; Jeantet et al. I.c. ; Martoja et al. I.c. ; ChassardBouchaud I.c. ; Lyon et Simkiss, I.c.) et sa présence doit être reliée à celle d'arylsulfatases. Celles-ci hydrolysent normalement des substrats arylsulfatés mais peuvent aussi fonctionner comme transférases (cf. Arnaud et al., l.c.) ; dans ce cas, des sul- 
foconjugaisons - considérées comme des processus de détoxication (El Mamlouk et Gessner, 1978) - de substances phénoliques ou de mucopolysaccharides d'origine endo- ou exogène peuvent se réaliser grâce à ces enzymes. Ainsi les corps denses apparaissent comme des complexes organiques très osmiophiles, renfermant une fraction protéique et sans doute une fraction MPS résultant de la transformation de substrats vacuolaires; ils correspondent à des éléments d'excrétion du métabolisme digestif. La présence d'aluminium renforce cette idée car ce métal, d'origine exogène, dont la toxicité est bien connue chez l'homme, est séquestré dans des inclusions lysosomales et des sphérocristaux (ChassardBouchaud et Galle, I.c. : Galle, 1986 : Humbert, I.c. ; Martoja et Martoja, I.c.). De plus, l'extrusion des cellules $B$ âgées dans la lumière intestinale libère les corps denses dont le matériel se retrouve au niveau des pelotes fécales en formation dans l'intestin moyen postérieur. Ceci explique d'une part les réactions histochimiques fortement positives de MPS au niveau de l'intestin postérieur où ce matériel est concentré (Arnaud et al., l.c.), d'autre part la détection des mêmes éléments minéraux dans le contenu intestinal postérieur et les cellules B. Notons que ces éléments doivent contribuer à l'élévation de la densité du contenu fécal observée par Nott et al. (1985), donc accélérer la sédimentation des pelotes fécales expulsées et mises en suspension dans le milieu.

En conclusion, nos résultats soulignent l'originalité des cellules $\mathrm{B}$ de Centropages typicus, et sans doute de tous les calanoides, dans la cytophysiologie digestive de ces copépodes : à une fonction de digestion intracellulaire, en relation avec l'intensité de la pinocytose et le développement de l'appareil vacuolaire, s'ajoute un rôle d'excrétion et de détoxication attesté par la présence d'aluminium et de substrats sulfatés dans des structures lysosomales.

Reçu en octobre 1986. Accepté en avril 1987.

\section{Références}

AL-MOHANNA S. Y., NOTT J. A., LANE D. J. W., 1985. Mitotic E- and secretory F-cells in the hepatopancreas of the shrimp Penaeus semisulcatus (Crustacea, Decapoda). J. mar. biol. Ass. U. K., 65, 901-910.

AL-MOHANNA S. Y., NOTT J. A., 1986. B- cells and digestion in the hepatopancreas of Penaeus semisulcatus (Crustacea : Decapoda). J. mar. biol. Ass. U. K., 66, 403-414.

ARNAUD J., BRUNET M., MAZZA J., 1978. Studies on the midgut of Centropages typicus (Copepod, Calanoid). I. Structural and ultrastructural data. Cell Tiss. Res., 187, 333-353.

ARNAUD J., BRUNET M., MAZZA J., 1980. Structure et ultrastructure comparées de l'intestin chez plusieurs espèces de copépodes calanoides (Crustacea). Zoomorphology, 95, 213-233.

ARNAUD J., BRUNET M., MAZZA J., 1981. La nutrition des copépodes pélagiques. Biol. Ecol. médit., 8, 87-97.

ARNAUD J., BRUNET M., MAZZA J., 1982. Etude de l'ovogenèse chez Centropages typicus (Copepoda, Calanoida). Reprod. Nutr. Dévelop., 22, 537-555. 
ARNAUD J., BRUNET M., MAZZA J., 1984. Cytochemical detection of phosphatase and arylsulphatase activities in the midgut of Centropages typicus (Copepod, Calanoid). Bas. Appl. Histochem., 28, 399-412.

CHASSARD-BOUCHAUD C., 1981. Rôle des lysosomes dans le phénomène de concentration du cadmium. Microanalyse par spectrographie des rayons X. C. R. Acad. Sci. Paris, 293, 261265.

CHASSARD-BOUCHAUD C., 1982. Localisation ultrastructurale du cadmium dans la glande digestive du crabe Carcinus maenas (Crustacé, Décapode). Microanalyse par spectrographie des rayons X. C. R. Acad. Sci. Paris, 294, 153-157.

CHASSARD-BOUCHAUD C., GALLE P., 1986. Bioaccumulation d'aluminium par les organismes marins. Mise en évidence par microscopie corpusculaire analytique. C. R. Acad. Sci. Paris, 302, 55-61.

DURFORT M., 1981. Mineral concretions in the intestinal epithelium of Cyclops strenuus Fisch. (Crustacea : Copepoda). Ultrastructural study. Bull. Inst. cat. Hist. nat., 47, 93-103.

DURFORT M., 1982a. Microanalisis de las concreciones intestinales de Cyclops strenuus Fisch. (Crustacea, Copepoda). Estudo preliminar. Misc. Zool., 6, 27-32.

DURFORT M., 1982b. Las concreciones minerales del hepatopancreas de Trachydermon cinereus, Thiele (Mollusca, Polyplacophora). Estudio ultrastructural. Iberus, 2, 1-17.

EL MAMLOUK T. H., GESSNER T., 1978. Carbohydrate and sulfate conjugations of p-nitrophenol by hepatopancreas of Homarus americanus. Comp. Biochem. Physiol., 61 C, 363-367.

GALLE P., 1986. La toxicité de l'aluminium. La Recherche, 178, 766-775.

GIBSON R., BARKER P. L., 1979. The decapod hepatopancreas. Oceanogr. mar. Biol., Ann. Rev., 17, 285-346.

GRAF F., MICHAUT P., 1977. Les sphérules calciques de l'épithélium caecal d'Orchestia (Crustacé, Amphipode), forme de transport de calcium dans le sens apico-basal. C. R. Acad. Sci. Paris, 284, 49-52.

HUMBERT W., 1978. Cytochemistry and X-ray microprobe analysis of the midgut of Tomocerus minor Lubbock (Insecta, Collembola) with special reference to the physiological significance of the mineral concretions. Cell Tiss. Res, 187, 397-416.

JEANTET A. Y., BALLAN-DUFRANÇAIS C., RUSTE J., 1980. Quantitative electron probe microanalysis on insects exposed to mercury. II. Involvement of the lysosomal system in detoxification processes. Biol. cell., 39, 325-334.

LOIZZI R. F., 1971. Interpretation of crayfish hepatopancreas function based on fine structure analysis of epithelial cell lines and muscular network. Z. Zellforsch., 113, 420-440.

LYON R., SIMKISS K., 1984. The ultrastructure and metal containing inclusions of mature cell types in the hepatopancreas of a crayfish. Tiss. Cell, 16, 805-817.

MARTOJA R., MARTOJA M., 1973. Sur les accumulations naturelles d'aluminium et de silicium chez quelques Invertébrés. C. R. Acad. Sci. Paris, 276, 951-954.

MARTOJA M., TAN TUE V., ELKAIM B., 1980. Bioaccumulation du cuivre chez Littorina littorea (L) (Gastéropode, Prosobranche) : signification physiologique et écologique. J. exp. mar. Biol. Ecol., 43, 251-270.

MASON A. Z., SIMKISS K., 1984. The ultrastructural localization of metals in specimens of Littorina littorea collected from clean and polluted sites. J. mar. Biol. Ass. U. K., 64, 699-720.

NOTT J. A., CORNER E. D. S., MAVLIN L. J., O'HARA S. C. M., 1985. Cyclical contributions of the digestive epithelium to faecal pellet formation by the copepod Calanus helgolandicus. Mar. Biol, 89, 271-279.

THIÉRY J. P., RAMBOURG A., 1974. Cytochimie des polysaccharides. J. Microsc., 21, 225-232. 